\title{
Is low-intensity shockwave therapy for erectile dysfunction ready for clinical practice?
}

\author{
Mikkel Fode ${ }^{1} \cdot$ Maarten Albersen $^{2} \cdot$ Peter Busch Østergren ${ }^{1}$
}

Received: 30 August 2018 / Accepted: 3 September 2018

(c) Springer Nature Limited 2018

Over recent years, Low-intensity Shockwave Therapy (LiESWT) has emerged as a potential cure for erectile dysfunction (ED) and this has created considerable excitement [1]. The predominate theoretical background for improvements in erectile function with Li-ESWT is that the treatment may promote the formation of new blood vessels and improve endothelial function in the corpora cavernosa [2]. However, in spite of promising pilot studies, the results of randomised controlled trials have been mixed with some finding effect on ED and some showing no differences between active treatment and sham control [3]. Therefore, Li-ESWT remains controversial.

In this month's issue of International Journal of Impotence Research, Yamaçake et al. [4] published the results of a randomised, double blinded, sham-controlled study investigating the effects of Li-ESWT for ED in Kidney Transplant Recipients [4]. This study is the first of its kind to evaluate the treatment in this specific group of patients. The study is also the first to investigate the specific LiESWT device used, the Dolorclast ${ }^{\circledR}$ Smart (Electro Medical Systems, Switzerland). The authors find an apparent effect of 6 treatments sessions with 7/10 patients in the treatment group experiencing an improvement of at least 5 points on the IIEF-5 scale vs. only $1 / 10$ in the sham group. At first glance this seems to confirm a possible effect of Li-ESWT and suggests that the treatment could be offered specifically to patients with kidney transplants. However, when examining the study closer, there may be reason for caution.

First of all, the authors included only 20 patients which is likely too few to evaluate the effects of a treatment for ED.

Mikkel Fode

mikkelfode@gmail.com

1 Department of urology, Herlev and Gentofte Hospital, Herlev, Denmark

2 Department of urology, University Hospitals Leuven, Leuven, Belgium
Thus, the study is by far the smallest RCT on the topic to date with previous power calculations showing that as many as $\mathbf{1 1 2}$ men are needed to show a clinically meaningful benefit compared to placebo [5]. In addition, there seem to be important differences between the groups. Most notably, the baseline IIEF-5 score is lower in the Li-ESWT group compared with the sham group (10.9 vs. 14.9). This is important because a given numeric improvement in the IIEF-5 score is likely to have less clinical meaning with low scores compared to higher scores [6]. In addition, statistically speaking, lower scores are more likely to spontaneously increase, while higher scores are more likely to decrease due to a concept termed "regression toward the mean" [7]. The finding that the changes in Erection Hardness Score (EHS) scores do not differ over time between the groups and the lack of difference in penile hemodynamic parameters should raise concerns that confounding factors may in fact have played a role. Other important factors are that the patients in the Li-ESWT group had their transplants shorter than the men in the sham group, which means that they may have adapted better to their new situation during the study and that more patients in the sham group suffered from diabetes. Even though these differences do not reach individual statistical significance, their combination may have had an influence in the small group of patients. In spite of these issues, the authors do not consider that their IIEF-5 findings may be due to confounding factors. Instead they question only the lack of improvements in EHS and hemodynamics, which skews their conclusions towards a positive appraisal of Li-ESWT.

The enthusiasm for Li-ESWT by Yamaçake et al. is understandable, as the treatment offers a potential cure for ED. This is something both patients and clinicians have been wanting for a long time [8]. However, such enthusiasm is problematic because it may cloud our judgement when designing trials and examining our clinical data. In this regard, a range of case series on Li-ESWT is often quoted when discussing the treatment. However, it is well known that the placebo effect in ED treatments may exceed 30\% 
[9]. This means that randomised, controlled trials (RCT) are an absolute necessity in this condition. In spite of this, such trials are only available for a minority of devices on the market and some are hampered by high risks of bias [3]. When examining only the RCTs with low to moderate risk of bias, one finds that three trials show a statistically significant improvement in IIEF scores compared to placebo [10-12], while another three fail to show such a difference $[5,13,14]$. In accordance with this, two out of three recent meta-analyses fail to find clinically meaningful improvements in erectile function with Li-ESWT compared to placebo [3]. With this in mind, it is prudent to be careful not to make too strong statements as to the effects of Li-ESWT in ED treatment. In the end, clinical research is simply about placing treatments into one of the following three categories: (1) Things which work so well that we should offer them to our patients, (2) Things which do not work and should not be offered to our patients, and (3) things which need more exploration before we potentially offer them to our patients. For now, Li-ESWT belongs to the third category and the Sexual Medicine community has an obligation to produce high quality research on the topic. Preferably, this should be in the form of multicenter RCTs. In addition to documenting the effects of Li-ESWT, such trials need to answer a multitude of questions over the coming years. These include determining the appropriate candidates and the optimal Li-ESWT protocol, assessing the long-term effects, and investigating if repeated treatments are needed.

In conclusion, Yamaçake et al. should be commended for contributing to our knowledge about Li-ESWT and their inclusion of a new device and a new patient group into the literature is valuable. However, the trial is not robust enough to make any definite conclusions and the authors are absolutely accurate in their final remark stating that "Additional studies, including large multicenter, longerterm, randomised, and sham-controlled studies, are required before Li-ESWT can be adopted as a standard therapy and a treatment that can "cure" ED." At this point in time, much research on Li-ESWT in ED is still needed.

\section{Compliance with ethical standards}

Conflict of interest The authors declare that they have no conflict of interest.

\section{References}

1. Fode M, Lowenstein L, Reisman Y. Low-Intensity extracorporeal shockwave therapy in sexual medicine: a questionnaire-based assessment of knowledge, clinical practice patterns, and attitudes in sexual medicine practitioners. Sex Med. 2017;5:e94.

2. Assaly-Kaddoum R, Giuliano F, Laurin M, Gorny D, Kergoat M, Bernabe J, et al. Low intensity extracorporeal shock wave therapy improves erectile function in a model of type ii diabetes independently of NO/cGMP pathway. J Urol. 2016;196:950.

3. Fode M, Hatzichristodoulou G, Serefoglu EC, Verze P, Albersen M. Low-intensity shockwave therapy for erectile dysfunction: is the evidence strong enough? Nat Rev Urol. 2017;14:593.

4. Yamaçake K, Carniero F, Cury J, Lourenco R, Piovesan A, Srougi $\mathrm{M}$, et al. Low-intensity shockwave therapy for erectile dysfunction in kidney transplant recipients. a prospective, randomized, double blinded, sham controlled study with evaluation by penile Doppler ultrasonography. Int J Impot Res. 2018. https://doi.org/10.1038/ s41443-018-0062-2.

5. Fojecki GL, Tiessen S, Osther PJ. Effect of low-energy linear shockwave therapy on erectile dysfunction-a double-blinded, sham-controlled, randomized clinical trial. J Sex Med. 2017; 14:106.

6. Rosen RC, Allen KR, Ni X, Araujo AB. Minimal clinically important differences in the erectile function domain of the International Index of Erectile Function scale. Eur Urol. 2011;60:1010.

7. Cutter, GR. Statistical cures and other fallacies. Mult Scler J. 2012;18:387-90.

8. Hanson-Divers C, Jackson SE, Lue TF, Crawford SY, Rosen RC. Health outcomes variables important to patients in the treatment of erectile dysfunction. J Urol. 1998;159:1541.

9. de Araujo AC, da Silva FG, Salvi F, Awad MC, da Silva EA, Damiao R. The management of erectile dysfunction with placebo only: does it work? J Sex Med. 2009;6:3440.

10. Vardi Y, Appel B, Kilchevsky A, Gruenwald I. Does low intensity extracorporeal shock wave therapy have a physiological effect on erectile function? Short-term results of a randomized, doubleblind, sham controlled study. J Urol. 2012;187:1769.

11. Kitrey ND, Gruenwald I, Appel B, Shechter A, Massarwa O, Vardi Y. Penile low intensity shock wave treatment is able to shift pde5i nonresponders to responders: a double-blind, sham controlled study. J Urol. 2016;195:1550.

12. Kalyvianakis D, Hatzichristou D. Low-intensity shockwave therapy improves hemodynamic parameters in patients with vasculogenic erectile dysfunction: a triplex ultrasonography-based sham-controlled trial. J Sex Med. 2017;14:891.

13. Olsen AB, Persiani M, Boie S, Hanna M, Lund L. Can lowintensity extracorporeal shockwave therapy improve erectile dysfunction? A prospective, randomized, double-blind, placebocontrolled study. Scand J Urol. 2015;49:329.

14. Yee CH, Chan ES, Hou SS, Ng CF. Extracorporeal shockwave therapy in the treatment of erectile dysfunction: a prospective, randomized, double-blinded, placebo controlled study. Int J Urol. 2014;21:1041. 